\title{
Electrophysiological correlates of crossmodal visual distractor congruency effects: Evidence for response conflict
}

\author{
Bettina Forster and Enea F. Pavone \\ City University, London, England
}

\begin{abstract}
To investigate the basis of crossmodal visual distractor congruency effects, we recorded event-related brain potentials (ERP) while participants performed a tactile location-discrimination task. Participants made speeded tactile location-discrimination responses to tactile targets presented to the index fingers or thumbs while ignoring simultaneously presented task-irrelevant visual distractor stimuli at either the same (congruent) or a different (incongruent) location. Behavioural results were in line with previous studies, showing slowed response times and increased error rates on incongruent compared with congruent visual distractor trials. To clarify the effect of visual distractors on tactile processing, concurrently recorded ERPs were analyzed for poststimulus, preresponse, and postresponse modulations. An enhanced negativity was found in the time range of the N2 component on incongruent compared with congruent visual distractor trials prior to correct responses. In addition, postresponse ERPs showed the presence of error-related negativity components on incorrect-response trials and enhanced negativity for congruent-incorrect compared with incongruent-incorrect trials. This pattern of ERP results has previously been related to response conflict (Yeung, Botvinick, \& Cohen, 2004). Importantly, no modulation of early somatosensory ERPs was present prior to the N2 time range, which may have suggested the contribution of other perceptual or postperceptual processes to crossmodal congruency effects. Taken together, our results suggest that crossmodal visual distractor effects are largely due to response conflict.
\end{abstract}

Investigations into the effects of events in one modality on processing of events in another modality and the integration of information across sensory modalities have recently gained increasing interest (see chapters in Calvert, Spence, \& Stein, 2004, and Spence \& Driver, 2004). Moreover, investigations into the interactions between visual and tactile events have revealed spatial constraints of peripersonal and extrapersonal space perception (for a review, see Maravita, Spence, \& Driver, 2003). Whereas tactile events define our proximal boundaries, visual events can occur close to the body surface or some distance away; furthermore, depending on the perceived distance of visual stimuli from tactile events, visual task-irrelevant stimuli may influence tactile discrimination judgments (for a review, see Spence, Pavani, Maravita, \& Holmes, 2004).

Specifically, one paradigm, the visual-tactile crossmodal distractor congruency task, has been used to investigate the multisensory construction of space (e.g., Maravita et al., 2003). In this paradigm, participants typically hold two cubes, one in either hand. One tactile stimulator and one light-emitting diode (LED) are placed in the upper and lower surface of each cube directly under or close to the index finger and thumb of each hand. On each trial, one tactile target and one visual distractor are presented simultaneously at any one of the four possible locations. The participants' task is to identify the location of the tactile target (top or bottom) while ignoring visual distractors. Visual distractors and tactile targets are presented from either the same location or different locations. Participants are typically slower and less accurate at discriminating the location of vibrotactile targets when simultaneously presented with a visual distractor from a different/incongruent location (i.e., tactile targets are presented at top locations and visual distractors at bottom locations, or vice versa) than they are when both tactile target and visual distractor are presented from the same/congruent location (i.e., either both from the same top location or both from the same bottom location). This difference in performance between incongruent and congruent trials is taken as an indication of visual distractor influence on tactile target discriminations.

Several explanations have been put forward to account for the influence of task-irrelevant visual events on tactile discriminations, as seen in the crossmodal congruency task. These explanations suggest either perceptual interactions between vision and touch, or higher cognitive processes as the bases of crossmodal congruency effects. To investigate a perceptual basis of crossmodal congruency effects, Spence, Pavani, and Driver (2004, Appendix) asked participants to perform as accurately as possible in a crossmodal congruency task. Under these unspeeded conditions, participants committed only a very low number of errors. These results suggest that the contribution of early 
perceptual interactions between vision and touch to crossmodal congruency effects are, if anything, minimal, since perceptual misplacement of tactile targets toward the location of visual distractors should be independent of time pressure (Spence, Pavani, \& Driver, 2004).

In addition to a perceptual explanation, two further explanations have been put forward to account for crossmodal visual distractor congruency effects. One is based on the assumption that visual distractors act as spatial attentional cues, shifting participants' attention to the visual distractor location, thus resulting in slower response times (RTs) and increased errors when the tactile targets and visual distractors are presented from incongruent locations. Likewise, several studies have also shown crossmodal congruency effects when visual distractors are presented before vibrotactile targets (Kennett, Spence, \& Driver, 2002; Shore, Barnes, \& Spence, 2006; Spence, Pavani, \& Driver, 2004) suggesting that visual distractors under these timing conditions may act as spatial attentional precues. According to the attentional-cue account of crossmodal congruency effects, tactile discrimination is facilitated when visual distractors and tactile targets are presented from the same location relative to when they are presented at different hands or sides. However, this account fails to explain the maximal interference effects of visual distractors when presented at the same hand or side as, but at a different location from, tactile targets. According to the spatial attentional-cue explanation, visual distractors close to the target location (e.g., at the same hand) should facilitate responses. However, stronger interference of distractors close to the target location is typically found.

The other postperceptual explanation is based on the assumption that crossmodal congruency effects reflect response conflict (Spence, Pavani, \& Driver, 2004; see also Shore et al., 2006; Shore \& Simic, 2005). This account explains the influence of incongruent visual distractors on tactile discriminations by means of inappropriate response priming of visual incongruent distractors, whereas congruent distractors facilitate responses by priming the appropriate response. In contrast to the attentional explanation, response conflict can account for the behavioral interference effect of visual distractors presented close to tactile targets but at a location associated with a different response.

According to the response conflict account of the crossmodal congruency task, both visual distractor and tactile targets activate separate response representations that are in conflict when distractor and target are presented at locations associated with different responses (e.g., target-attop and distractor-at-bottom locations). During performance of a task, cognitive control processes are thought to monitor and regulate ongoing processes in a goal-directed manner; this includes (1) detection of conflict during information processing, that may arise from activation of different response representations, and (2) detection and correction of error responses. Event-related brain potential (ERP) studies investigating the neural response of performance monitoring have typically investigated the neural response following error responses (Falkenstein, Hohnsbein, Hoormann, \& Blanke, 1991; Gehring, Goss,
Coles, Meyer, \& Donchin, 1993); recently, neural processes prior to response execution have also been linked to monitoring processes (Nieuwenhuis, Yeung, van den Wildenberg, \& Ridderinkhof, 2003; Yeung, Botvinick, \& Cohen, 2004). According to the conflict theory (Botvinick, Braver, Barch, Carter, \& Cohen, 2001), the performance monitoring system provides a continuous evaluation of the degree of conflict generated by the activation of competing response units. Furthermore, Yeung et al. suggested that neural responses both before and after response execution reflect the amount of conflict present; that is, conflict associated with correct responses is present before response execution, and conflict associated with erroneous responses is present afterward.

Following Yeung et al.'s (2004) account of conflict monitoring, we investigated whether electrophysiological correlates of response conflict are present in the crossmodal visual distractor congruency task; if so, due to the ambiguity of incongruent stimuli leading to increased conflict in selecting the appropriate response, incongruent visual distractors should induce stronger response conflict than congruent visual distractors, prior to the execution of correct responses. Likewise, electrophysiological studies of preresponse conflict have reported that the N2 component - present around $250 \mathrm{msec}$ after stimulus onset in stimulus-locked waveforms, and around $150 \mathrm{msec}$ prior to a response in response-locked waveforms - shows greater negativity on incongruent than on congruent trials (Kopp, Rist, \& Mattler, 1996; Liotti, Woldorff, Perez, \& Mayberg, 2000; Nieuwenhuis et al., 2003). Similarly, in the crossmodal visual distractor congruency task, conflict should be reflected in enhanced negativity of N2 components of ERPs when visual distractors and tactile targets are incongruent, rather than when they are presented from congruent locations.

After the execution of responses, conflict also should be stronger for erroneous responses on congruent than on incongruent trials. This assumption derives from the suggestion that we constantly monitor our actions and compare them to internal representation of correct responses. In the case of erroneous responses, a mismatch is detected which is reflected in the error-related negativity (ERN; Gehring et al., 1993; see also Falkenstein et al., 1991) present shortly after execution of erroneous responses. Originally, this component was thought to reflect the detection of error commission (Falkenstein et al., 1991; Gehring et al., 1993) but suggestions have recently been made to the effect that it reflects the amount of conflict present immediately after response execution, and is closely related to error-correcting activity (Botvinick et al., 2001; Yeung et al., 2004). Therefore, after an erroneous response, conflict between the representation of the correct response and the actual incorrect response should be present; furthermore, this conflict should be stronger for erroneous responses on congruent trials than for those on incongruent trials. Therefore, response conflict should be strongest immediately following an incorrect response, when visual distractors are presented from the same congruent location as tactile targets. This is due to conflict between the equal activations of response representations 
by visual distractors and tactile targets that are both in conflict with the actual incorrect response. In contrast, on incongruent visual distractor trials, only tactile targets activate response representations that are different from the actual incorrect response, thus leading to less response conflict than on congruent visual distractor trials.

To investigate the hypothesis that crossmodal visual distractor congruency effects reflect response conflict, we measured participants' behavioral performance in tactile location discriminations ("upper" at the index finger vs. "lower" at the thumb), while ignoring visual distractors presented from the same location, or a different one, on the same hand (e.g., both at the index finger, or one at the index finger and the other at the thumb). ${ }^{1}$ Concurrently recorded ERPs were analyzed in the time range of the N2 and ERN components. According to the conflict-monitoring theory, we expect to find correlates of response conflict activity on correct trials prior to (i.e., N2 time range) and on error trials following (i.e., presence of ERN) response execution. In addition, we also analyzed early somatosensory components to investigate the hypothesis that perceptual or attentional orienting processes were the bases of the crossmodal visual distractor congruency effect. Attentional modulations have repeatedly been reported to enhance the N140 component (e.g., Desmedt \& Robertson, 1977; Eimer \& Forster, 2003; Forster \& Eimer, 2004, 2005a; García-Larrea, Lukaszewicz, \& Mauguière, 1995; Michie, Bearpark, Crawford, \& Glue, 1987), although a study by Schürmann, Kolev, Menzel, and Yordanova (2002) found that perceptual interaction between vision and touch results in modulations of early somatosensory components already starting in the time range of the P100 component. Therefore, with respect to stimulus-locked ERP components, we analyzed - in addition to the longer-latency N2 component, which has been related to response conflictearly somatosensory components present around $100 \mathrm{msec}$ (P100) and $140 \mathrm{msec}$ (N140) after stimulus onset, to investigate early perceptual and attentional effects of visual distractors on tactile processing.

\section{METHOD}

\section{Participants}

Twelve volunteers ( 5 males and 7 females) with a mean age of 28 years (age range, 21-37 years) participated in the experiment. All participants were naive regarding the purpose of the experiment. According to self-report, all were right-handed and had normal or correctedto-normal vision, and all reported having normal touch. Participants gave their written informed consent prior to their participation.

\section{Apparatus, Stimuli, and Procedure}

Participants sat at a table in a dimly lit room, with their forearms placed comfortably on the tabletop in front of them, and holding a wooden cube $(7 \mathrm{~cm}$ high $\times 3 \mathrm{~cm}$ wide $\times 5 \mathrm{~cm}$ deep) between the thumb and index finger of each hand. Between the cubes, a $20-\mathrm{cm}$ wide piece of cardboard was placed at a $45^{\circ}$ angle on the table surface. A black fixation cross was drawn on the center of the cardboard and the midline of the cubes was aligned with the horizontal line of the fixation cross. Participants were asked to tilt the cubes so that the side facing the participant was parallel to the cardboard surface. Each cube had two solenoids embedded, one in the lower surface and one in the upper, placed directly under the thumb and index finger of each hand. Tactile stimuli were presented using $12-\mathrm{V}$ solenoids, driving a metal rod with a conical tip to the finger pad, making contact with the fingers whenever a current was passed through the solenoid. Tactile target stimuli consisted of one rod contacting a finger for $80 \mathrm{msec}$; this gave rise to a suprathreshold touch sensation. Four red LEDs (5 $\mathrm{mm}$ in diameter) were also mounted on the cubes, two on each, to provide the visual distractor stimuli; one was placed next to each of the four tactile stimulators. Visual distractor stimuli consisted of the illumination of one LED for $80 \mathrm{msec}$. On each trial, one tactile target and one visual distractor stimulus were presented simultaneously from the same cube (both from either the left or the right cube) at either the same location (both at either the upper or lower cube side) or at opposite locations (one at the upper cube side, the other at the lower). Participants responded by stepping on one of two adjacent pedals. White noise was presented from a loudspeaker directly in front of the participants to mask any sounds made by the operation of the solenoids.

Fifteen experimental blocks, with 64 trials per block, were run. A block of 64 practice trials was given before the start of the experiment. The practice trials were excluded from the final analysis. On 32 trials of each block, one target and one distractor were presented from the same location (congruent trials), and on the remaining 32 trials, target and distractor were presented from opposite locations (incongruent trials). Congruent and incongruent trials were presented with equal probability from the left and right cube. Participants were instructed to make speeded location discriminations to tactile targets by pressing one of two pedals. Half of the participants pressed the right pedal when the target appeared at upper locations and the left pedal to indicate lower locations. For the remaining participants, this association between target location and response foot was reversed. If no response was made within $700 \mathrm{msec}$ of target onset, an acoustic feedback $(1000 \mathrm{~Hz})$ was presented for $500 \mathrm{msec}$ before the start of the next trial. The intertrial interval was $620 \mathrm{msec}$.

\section{Data Acquisition and Analysis}

Electroencephalograph (EEG) interaction was recorded with $\mathrm{Ag}-$ $\mathrm{AgCl}$ electrodes and linked-earlobe reference ${ }^{2}$ from Fp1, Fp2, F3, F4, C3, C4, P3, P4, O1, O2, F7, F8, T7, T8, P7, P8, Fz, FCz, Cz, Pz, $\mathrm{FC} 1, \mathrm{FC} 2, \mathrm{CP} 1, \mathrm{CP} 2, \mathrm{FC} 5, \mathrm{FC} 6, \mathrm{CP} 5$, and CP6 (according to the 10-20 system). Horizontal EOG (HEOG) was recorded bipolarly from electrodes positioned on the outer canthii of both eyes. A BrainAmps amplifier system and Brain Vision Recorder and Analyzer 1.05 software (Brain Products, $\mathrm{GmbH}$ ) were used for recording and offline analysis of the EEG data. Impedance was kept below $2 \mathrm{~K} \Omega$ for reference and ground electrodes and below $5 \mathrm{~K} \Omega$ for all other electrodes; amplifier band-pass was 0.01 to $100 \mathrm{~Hz}$, and digitization rate was $500 \mathrm{~Hz}$. EEG and HEOG were epoched in separate offline analyses. To investigate stimulus-locked ERPs, epochs were extracted for a period starting $200 \mathrm{msec}$ prior until $800 \mathrm{msec}$ after to the onset of the visual and tactile stimuli. To investigate response-locked ERPs, epochs were extracted for a period from $800 \mathrm{msec}$ prior until $100 \mathrm{msec}$ after a response ("preresponse ERPs"), and for a period from $200 \mathrm{msec}$ prior until $600 \mathrm{msec}$ after the onset of the participants' responses ("postresponse ERPs"). Trials with horizontal eye movements, eye blinks, or any other artifact, and trials with RTs below $100 \mathrm{msec}$ and above $1,000 \mathrm{msec}$, were excluded separately for each epoch type.

Stimulus-locked ERPs were averaged relative to a $200-\mathrm{msec}$ prestimulus baseline for target-distractor congruency and response accuracy resulting in four averages [condition (mean number of trials; range)]: congruent-correct trials $(407 ; 234-456)$; congruent-incorrect trials $(37 ; 11-163)$; incongruent-correct trials (339; 94-429); and incongruent-incorrect trials (86; 38-160). Postresponse ERPs were also averaged relative to a $200-\mathrm{msec}$ preresponse baseline on the basis of target-distractor congruency and response accuracy, resulting in four different averages: congruentcorrect trials $(313 ; 39-451)$; congruent-incorrect trials $(30 ; 1-149)$; incongruent-correct trials $(249 ; 24-420)$; and incongruent-incorrect trials $(54 ; 5-138)$. All averages were then filtered with a low-pass digital filter (zero-phase shift type) of $30 \mathrm{~Hz}$.

To further explore N2 effects, difference waveforms of stimuluslocked ERPs were calculated by subtracting ERP waveforms elic- 
ited on congruent-correct trials from ERP waveforms elicited on incongruent-correct trials. In addition, preresponse ERPs elicited on incongruent-correct $(285 ; 94-424)$, congruent-correct (353; $195-461)$, and error $(106 ; 12-168)$ trials were averaged relative to a baseline taken from 800 to $700 \mathrm{msec}$ prior to the response and were filtered with a low-pass digital filter of $30 \mathrm{~Hz}$. In addition, a highpass filter with a low cutoff of $2 \mathrm{~Hz}$ was applied to both difference waveforms and preresponse ERPs (further exploring N2 effects), to remove the contribution of slow parietal positivities that may mask the frontocentral negativities of interest (see, e.g., Yeung et al., 2004).

ERP mean amplitudes were computed for each participant for the averages of response-locked ERPs for the time window of the N2 component ( -180 to $-80 \mathrm{msec}$ prior to the onset of participants' responses in preresponse ERPs) and the ERN component (20-120 msec following the onset of participants' responses in postresponse ERPs), and for the averages of stimulus-locked ERPs for the time window of the somatosensory P100 (80-128 msec), N140 (130-174 msec), and the N2 component (210-310 msec; all time windows following the onset of simultaneously presented tactile and visual stimuli).

Statistical analyses were conducted for midline electrode sites ( $\mathrm{Fz}, \mathrm{FCz}, \mathrm{Cz}$, and $\mathrm{Pz}$ ) to investigate the $\mathrm{N} 2$ and $\mathrm{ERN}$ components, and for lateral electrode sites (F3, F4, FC5, FC6, C3, C4, CP5, CP6, $\mathrm{P} 3$, and $\mathrm{P} 4)$ over the left and right hemispheres close to and over the somatosensory cortex to investigate modulations of the early P100 and N140 somatosensory components. Separate ANOVAs were conducted for mean amplitude values in the time range of the P100 (80-128 msec post-stimulus-onset), N140 (130-174 msec poststimulus-onset), N2 (210-310 msec post-stimulus-onset, and - 180 to $-80 \mathrm{msec}$ pre-response-onset), and ERN (20-120 msec postresponse-onset) components with factors visual distractor location (congruent vs. incongruent), response type (correct vs. incorrect), and electrode $(\mathrm{Fz}, \mathrm{Cz}, \mathrm{FCz}$, and $\mathrm{Pz}$ ) for midline electrode site analyses, or electrode $(\mathrm{F} 3 / 4, \mathrm{FC} 5 / 6, \mathrm{C} 3 / \mathrm{C} 4, \mathrm{CP} 5 / \mathrm{CP} 6$, and P3/P4) and hemisphere (ipsilateral vs. contralateral to the side of stimulation) for lateral electrode site analyses. Mean amplitude ERP values and behavioral data were analyzed using SPSS (Version 11.1) general linear model (GLM) software with the Greenhouse-Geisser correction for nonsphericity, applied where appropriate.

\section{RESULTS}

\section{Behavioral Results}

Trials on which participants made a premature response (RT $<100 \mathrm{msec}$ ) or failed to respond within $1,000 \mathrm{msec}$ were removed from both RT and accuracy analyses. This resulted in the removal of an average of $3.45 \%$ of trials across all participants. Accuracy and mean RTs of correct responses were analyzed separately, using a repeated measures ANOVA with factors tactile target location (up vs. down) and visual distractor congruency (congruent vs. incongruent). Table 1 shows average RTs and error rates separately for trials when visual distractors and tactile targets were congruent and incongruent. Participants responded on average $68 \mathrm{msec}$ faster and committed fewer mistakes when visual distractors were congruent with tactile stimuli. Statistical analysis showed a main effect of visual distractor congruency for RTs [469 msec compared with $537 \mathrm{msec} ; F(1,11)=75.77, p<.0001]$ and error rates $[24 \%$ compared with $9 \% ; F(1,11)=7.76, p=.02]$.

\section{ERP Results}

To investigate modulations of early somatosensory components (P100 and N140) and components associated with response conflict (N2 and ERN), poststimulus
Table 1

Mean Response Times (RTs, in Milliseconds, With Standard Deviations) and Percentages of Error ( $\% E$ ) for Tactile Iarget Location Discriminations, Shown Separately for Congruent and Incongruent Visual Distractor Trials

\begin{tabular}{|c|c|c|c|}
\hline \multirow{2}{*}{$\begin{array}{c}\text { Visual Distractor } \\
\text { Location }\end{array}$} & \multicolumn{2}{|c|}{ RTs } & \multirow[b]{2}{*}{$\% \mathrm{E}$} \\
\hline & $M$ & $S D$ & \\
\hline Congruent & 469 & 15.73 & 9 \\
\hline Incongruent & 537 & 16.65 & 24 \\
\hline
\end{tabular}

(P100, N140, and N2), preresponse (N2), and postresponse (ERN) ERP waveforms were analyzed.

Poststimulus ERPs. Figure 1 shows stimulus-locked ERPs elicited by congruent (solid lines) and incongruent (dashed lines) visual distractor and tactile target stimuli prior to the execution of correct responses. Starting around the onset of the $\mathrm{N} 2$ component at about $200 \mathrm{msec}$ after stimulus onset, an enhanced negativity is present on incongruent rather than congruent trials, and no modulations are present prior to this. Statistical analysis showed no significant main effect of or interaction involving the factors visual distractor location or response type in the time range of the P100 or N140 components. For the following analysis window of the $\mathrm{N} 2$ component, a main effect of visual distractor location $[F(1,11)=13.11$, $p<.01]$ and a visual distractor location by response type interaction $[F(1,11)=10.34, p<.01]$ was present. Follow-up analysis separate for correct- and incorrectresponse trials showed a significant visual distractor location effect only for correct-response trials $[F(1,11)=$ 22.77, $p<.01$ for correct-response and $F(1,11)<1$, n.s., for incorrect-response trials], confirming enhanced N2 amplitudes when visual distractors were incongruent with tactile target locations prior to correct responses.

To further explore the enhancement of ERPs on incongruent-correct compared with congruent-correct trials, difference waveforms were calculated by subtracting ERPs on congruent-correct from incongruent-correct trials. Figure 2 shows the topographic distribution of the enhancement of incongruent-correct trials that was centered over frontocentral electrode sites for the time range of the N2 component (Figure 2A: $210-310 \mathrm{msec}$ after stimulus onset). In contrast, for the subsequent time window (Figure 2B: 330-430 msec after stimulus onset) the difference in ERP waveforms between incongruent- and congruent-correct trials was more pronounced over posterior electrode sites.

Preresponse ERPs. Figure 3 shows the relative timing of the N2 and the ERN in response-locked ERPs: Figure $3 \mathrm{~A}$ shows ERP waveforms elicited on incongruentcorrect (solid line), congruent-correct (dashed line), and on incorrect-response (dashed-dotted line) trials at electrode FCz. The N2 is clearly visible on correct-response trials prior to response onset, whereas the ERN is present following response onset. ERP amplitudes were more negative on incongruent- than on congruent-correct trials starting around $200 \mathrm{msec}$ prior to the response. Statistical analysis of correct-response trials with the factors visual distractor location and electrode showed a main ef- 


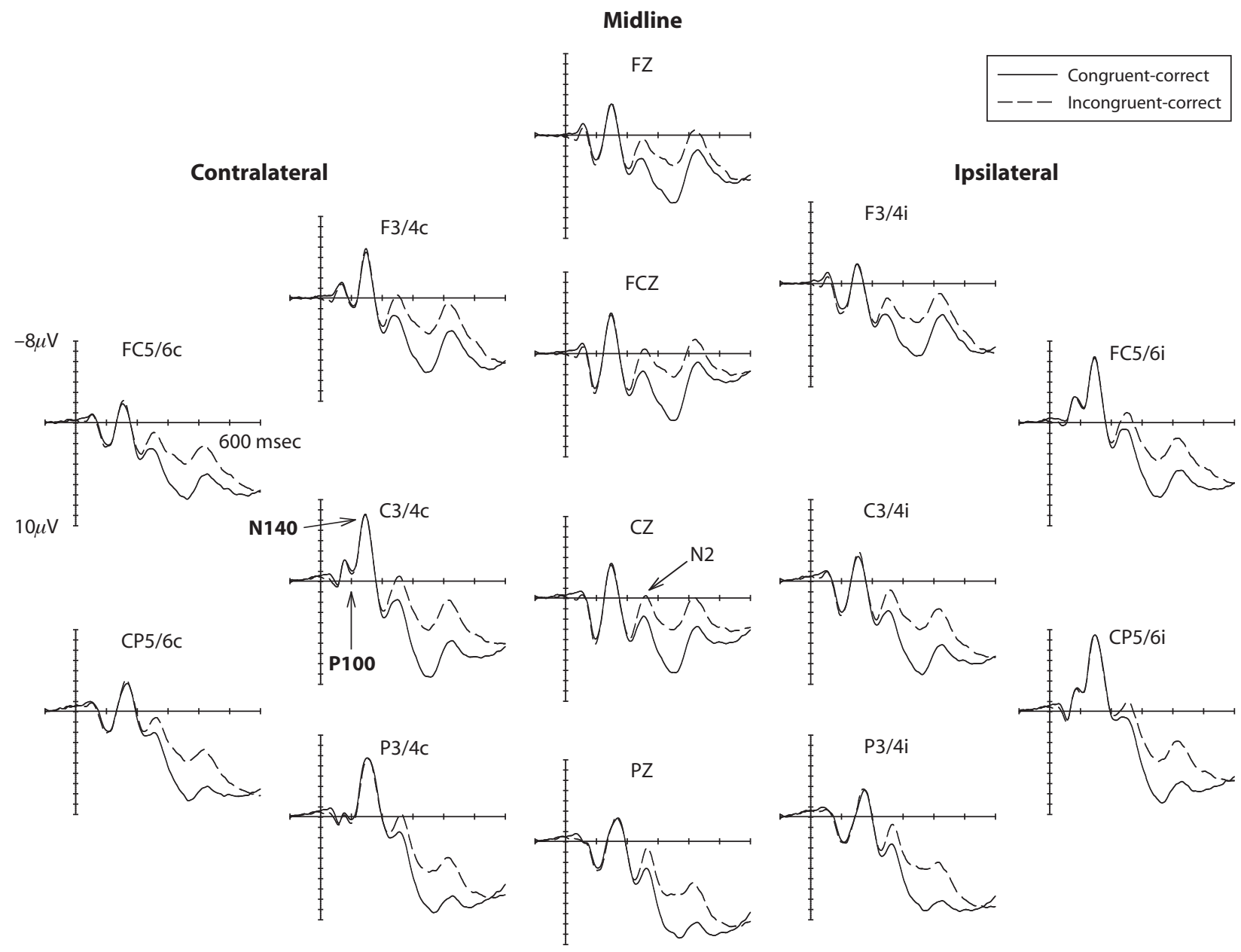

Figure 1. Grand-averaged ERPs in response to congruent (solid lines) and incongruent (dashed lines) visual distractor and tactile target stimuli in the 600 msec following stimuli onset prior to correct responses. ERPs are shown at electrode sites over the hemisphere contralateral and ipsilateral to the stimulated hand and at midline electrodes.

fect of visual distractor location $[F(1,11)=13.00, p<$ $.01]$ confirming the presence of an enhanced negativity on incongruent-correct trials, also in response-locked ERPs. To further explore the topographic distribution of the N2 modulation present in response-locked ERPs, dif- ference waveforms were calculated by subtracting ERPs on congruent-correct from ERPs on incongruent-correct trials. Figure 3B shows a frontocentral distribution of the $\mathrm{N} 2$ modulation (left side), similar to the topographic distribution seen after response execution (ERN; right side).
A

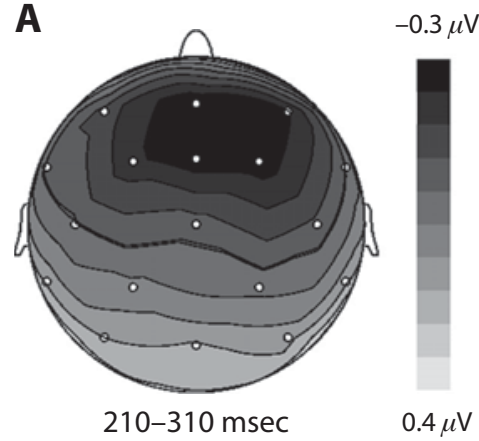

B

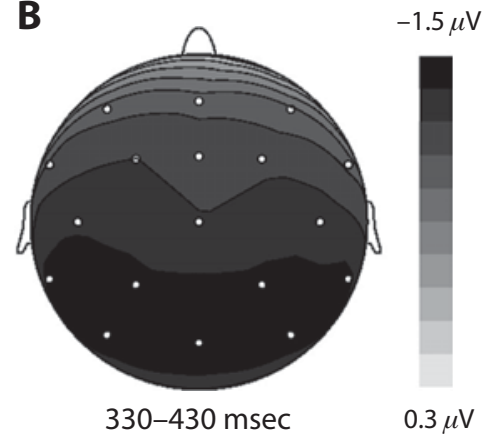

Figure 2. Scalp voltage maps of the difference of stimulus-locked ERP waveforms elicited on congruent-correct trials subtracted from ERP waveforms elicited on incongruent-correct trials. (A) Time range of the $\mathrm{N} 2$ effect, $210-310 \mathrm{msec}$ poststimulus. (B) Subsequent time range, 330-430 msec poststimulus. 
Postresponse ERPs. Figure 3A shows responselocked ERP waveforms on congruent- (dashed line) and incongruent-incorrect (dashed-dotted line) response trials, and on correct-response trials averaged across incongruent and congruent visual distractor trials (solid line). ERP amplitudes are more negative on incorrect-response than on correct-response trials, with ERN components clearly present on incorrect-response trials immediately following response execution. Furthermore, ERN amplitudes are more negative on congruent-incorrect than on incongruent-incorrect response trials. These observations were substantiated by statistical analysis that showed a main effect of response type $[F(1,11)=17.18, p<.01]$ confirming the presence of ERN components with more negative ERP amplitudes on incorrect-response than on correct-response trials, and a main effect of visual distractor location $[F(1,11)=9.02, p<.02]$ reflecting larger ERP amplitudes when visual distractors were congruent than when they were incongruent with tactile targets. In addition, a main effect of electrode $[F(3,33)=15.29, p<.001]$ and a significant electrode $\times$ response type interaction $[F(3,33)=6.20, p<.01]$ were present. Follow-up analyses separate for each electrode showed significantly more negative ERP amplitude values on incorrect-response than on correct-response trials at all electrodes [all $t(1,11)>$ $3.18, p<.01]$. To further investigate the effect of visual distractor location, two one-way ANOVAs were performed separately for correct- and incorrect-response trials with factors visual distractor location and electrode. This analysis showed a main effect of visual distractor location on incorrect-response trials only $[F(1,11)=8.95, p<.02$; $F(1,11)=4.17$, n.s., on correct-response trials] confirming that visual distractor congruency effects on ERN components were only elicited following incorrect responses.

A

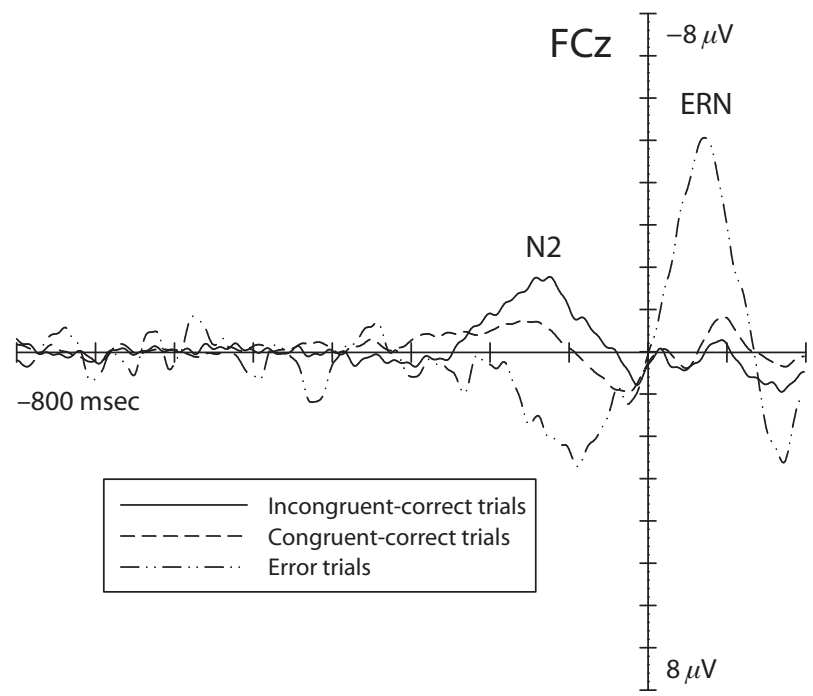

B

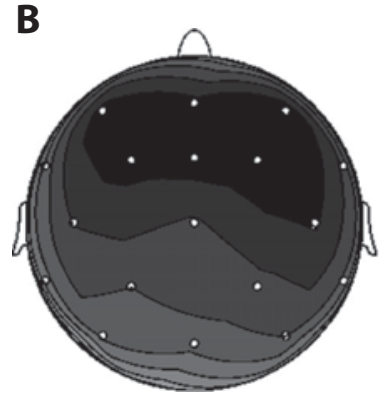

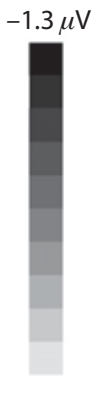

$-0.5 \mu \mathrm{V}$

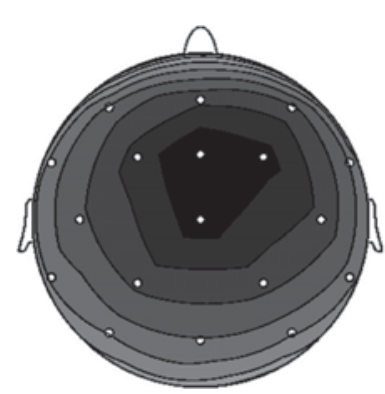

$0.9 \mu \mathrm{V}$

Figure 3. (A) Grand-averaged ERPs elicited on correct-response trials separate for congruent (solid line) and incongruent (dashed line) visual distractor trials, and on erroneous-response trials (dotted-dashed line) in the $800 \mathrm{msec}$ prior to, and the 200 msec following, a response at electrode FCz. (B) Scalp voltage maps for the time point of the $\mathbf{N} 2$ component $(-126-m s e c$ preresponse; left side) and the ERN (76-msec postresponse; right side). The left map shows the difference of ERP waveforms elicited on congruent-correct trials subtracted from ERP waveforms elicited on incongruent-correct trials; the right map shows ERPs elicited on erroneous response trials averaged across congruent and incongruent visual distractor trials. 


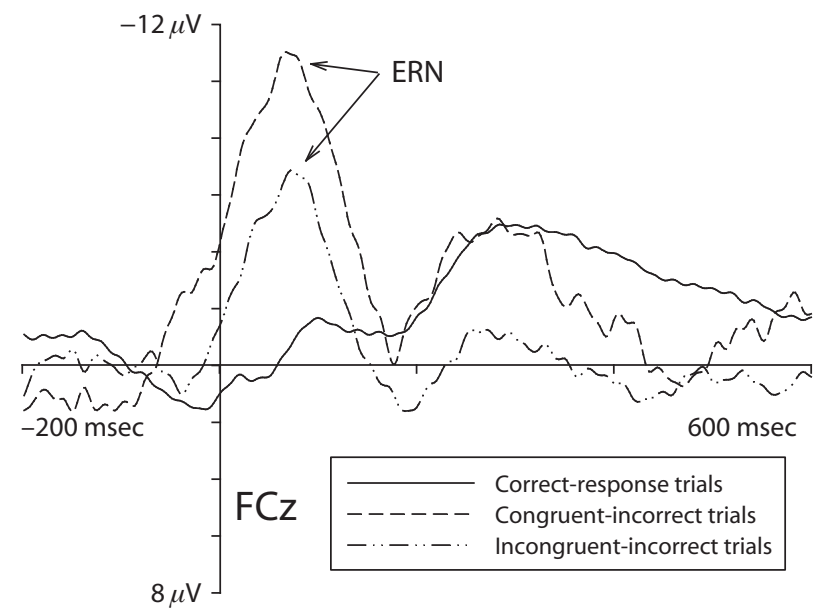

Figure 4. Grand-averaged ERPs elicited after correct responses (solid line) and erroneous responses separate for congruent (dashed line) and incongruent (dashed-dotted line) visual distractor trials in the $600 \mathrm{msec}$ following response onset at electrode FCz. ERPs on correct-response trials are averaged across congruent-correct and incongruent-correct trials.

\section{DISCUSSION}

In the present study, ERP markers of response conflict were evaluated in a crossmodal visual distractor congruency task. Participants performed a tactile location (up or down) discrimination task, while ignoring visual distractors delivered at either the same location as tactile targets (congruent) or at opposite locations (incongruent). In line with previous behavioral findings (Shore et al., 2006; for a review, see Spence, Pavani, \& Driver, 2004), participants were faster and more accurate when tactile targets and visual distractors were congruent than when these were incongruent, suggesting that the location of the task-irrelevant distractor lights has a profound effect on location judgments of tactile target stimuli. Furthermore, electrophysiological correlates of response conflict were present before and after response execution, suggesting that task-irrelevant visual stimuli induced response conflict when presented from locations incongruent to those of tactile targets.

We found that electrophysiological correlates of stimulus processing showed enhanced negativity, for incongruent compared with congruent visual distractor trials, that was present prior to correct-response execution, starting in the time range of the N2 component. Likewise, previous studies, investigating ERP correlates of response conflict, have reported modulation of the N2 component on correct-response trials when response incongruent distractors were present (Davies, Segalowitz, Dywan, \& Pailing, 2001; Nieuwenhuis et al., 2003; Yeung et al., 2004). We found an enhanced negativity for incongruent-correct compared with congruent-correct trials to be present in the time range of the $\mathrm{N} 2$ in stimulus-locked waveforms. The difference in stimulus-locked ERP waveforms between incongruent- and congruent-correct trials was largest over frontocentral electrode sites for the time range of the N2, although this difference showed a posterior distribution for later latencies. An enhanced negativity for incongruent- compared with congruent-correct trials was also present prior to response execution in responselocked ERPs, and the difference of these waveforms revealed again a frontocentral distribution of the N2 modulation. Our results suggest, therefore, that, prior to correct tactile location discriminations, simultaneously presented task-irrelevant visual distractors induce response conflict when presented from a location associated with a different response. In contrast, after response execution no reliable difference between incongruent-correct and congruent-correct trials was present, suggesting the presence of preresponse conflict on correct-response trials that diminishes after response execution (see Yeung et al., 2004).

In addition to the presence of preresponse conflict as reflected in modulations of the $\mathrm{N} 2$ component, we also report the presence of ERN components immediately following incorrect-response execution. The presence of ERN components only after erroneous responses is in line with previous studies suggesting that this component is related to online monitoring of responses reflecting conflict detection between the representation of the correct response and the actual incorrect response (Botvinick et al., 2001; Yeung et al., 2004). Furthermore, we found ERNs to be larger on congruent than on incongruent trials, in line with the prediction that postresponse conflict after an incorrect response is larger on trials when both tactile target and visual distractor are presented from congruent than from incongruent locations. These findings are consistent with results of a simulation study of the connectionist model of conflict monitoring (Yeung et al., 2004), suggesting stronger response conflict after incorrect responses resulting from greater activation of correctresponse representations when both distractor and target are congruent than when they are incongruent.

Both the N2 and ERN components have been suggested to reflect conflict monitoring processes. In line with previous studies, we also found that both components show similar topographic distributions (Figure 3B), and it has been suggested that both share a neural source located in the anterior cingulate cortex (e.g., Dehaene, Posner, \& Tucker, 1994; Holroyd, Dien, \& Coles, 1998; Nieuwenhuis et al., 2003; van Veen \& Carter, 2002). Furthermore, enhanced N2 components by stimuli associated with incongruent responses prior to correct responses and enhanced ERN components following erroneous responses have been observed in situations characterized by highresponse conflict like the flanker (Kopp et al., 1996; Yeung et al., 2004), Stroop (Gehring et al., 1993; Liotti et al., 2000), and go/no-go (Nieuwenhuis et al., 2003) tasks. Likewise, we observed a similar pattern of modulations of the N2 and ERN components in the crossmodal congruency task, suggesting that task-irrelevant visual distractors induce response conflict when presented close to tactile target locations but at a location associated with a different response.

Attentional modulations of tactile processing are typically seen in enhancement of the somatosensory N140 component followed by a sustained negativity for later latencies (e.g., Desmedt \& Robertson, 1977; Eimer \& Forster, 2003; 
Eimer, Forster, Fieger, \& Harbich, 2004; Forster, Eardley, \& Eimer, 2007; Forster \& Eimer, 2004, 2005a; GarcíaLarrea et al., 1995; Michie et al., 1987; Van Velzen, Forster, $\&$ Eimer, 2002). If visual distractors were acting as attention directing cues in the crossmodal congruency task, the strongest cuing effects would be seen when visual distractors and tactile targets were presented at the same location. This would be reflected in an enhanced negativity for stimuli presented at congruent rather than incongruent locations. However, no modulations of early somatosensory ERPs dependent on visual distractor congruency were present in the time range of the N140 component. Moreover, an enhanced negativity was present at later latencies, but this reflected enhanced processing when visual distractors were presented at locations incongruent to tactile targets. Taken together, our results do not support the attentional cue account of visual distractor congruency effects; rather, our electrophysiological results are in line with the response conflict account.

In the present study, visual distractors were only presented from locations on the same hand as tactile targets, since under these conditions visual distractor effects are maximal. Therefore, in the present study, one condition commonly included in a visual-tactile crossmodal distractor congruency task was excluded. Including such a condition could have made it easier to observe crossmodal attentional facilitation effects. In this condition, visual distractor and tactile target are presented at opposite sides (e.g., tactile target at the left hand; visual distractor at the right hand). Following the attentional explanation of crossmodal congruency effects, visual distractors may act as exogenous attentional cues that facilitate tactile target processing when presented from the same location. Therefore, by comparing ERPs elicited on trials when visual distractors are presented from the same location as tactile targets with ERPs elicited on trials when tactile target and visual distractor are presented from opposite sides, crossmodal exogenous attentional effects on early somatosenory processing could be revealed.

The conflict monitoring theory (Botvinick et al., 2001; Yeung et al., 2004) states that conflict occurs when two or more incompatible response representations are activated. In the present study, participants performed speeded tactile discriminations in a crossmodal visual distractor congruency task, in which visual distractors were presented from either congruent or incongruent locations on the same hand. Previous behavioral studies have typically included a condition in which incongruent visual distractors are presented from an incongruent location opposite the tactile target location (e.g., visual distractor, bottom left hand; tactile target, top right hand). Under this condition, visual distractor effects on tactile location-discrimination are attenuated, compared with conditions under which visual distractors are presented from an incongruent location on the same hand as the tactile target (e.g., Spence, Pavani, \& Driver, 2004; Spence, Pavani, et al., 2004). Therefore, visual distractors, especially those at a response-incongruent location close to (or perceived as being close to) the tactile target site, interfere adversely with tactile target location discriminations (for a review, see Spence, Pavani, \& Driver, 2004). Under both conditions (same vs. different hand), visual distractors are presented from an incongruent location and-according to the response conflict account - should induce similar amounts of conflict. Therefore, the difference in visual distractor effects was found to be dependent on the proximity of visual distractor and tactile target locations. This may suggest that additional perceptual interaction between visual distractors and tactile targets takes place when both stimuli are presented at the same hand. However, whereas visuotactile interactions have been reported to be reflected in modulations already present in the time range of the early somatosensory P100 component (Schürmann et al., 2002), for spatially congruent as opposed to incongruent visuotactile stimuli, we found modulation only of longer latency components. ${ }^{3}$ Longer-latency modulations have been related to postperceptual processes, reflecting in-depth stimulus processing of task-relevant stimuli (Michie, 1984).

In sum, in the present study behavioral and electrophysiological correlates of poststimulus and postresponse processing were evaluated in a crossmodal congruency task. Participants' RTs were slower and error rates increased when task-irrelevant visual distractors were presented from locations incongruent to tactile targets. Furthermore, concurrently recorded ERPs showed enhanced negativity of the N2 component for incongruent-correct compared with congruent-correct response trials; in addition, postresponse ERNs were enhanced on congruent error trials. This pattern of ERP results has previously been related to the presence of preresponse and postresponse conflict (Yeung et al., 2004). Taken together, our results suggest that crossmodal visual distractor congruency effects are largely due to response conflict.

\section{AUTHOR NOTE}

E.F.P. is now at Fondazione "Santa Lucia" IRCCS, Rome, Italy, and the Department of Psychology, University "La Sapienza," Rome, Italy. This research was supported by a grant from the Biotechnology and Biological Research Council (BBSRC). Correspondence concerning this article should be addressed to B. Forster, Department of Psychology, City University, London EC1V0HB, England (e-mail: b.forster@city.ac.uk).

Note-This article was accepted by the previous editorial team, when John Jonides was Editor.

\section{REFERENCES}

Botvinick, M. M., Braver, T. S., Barch, D. M., Carter, C. S., \& Cohen, J. D. (2001). Conflict monitoring and cognitive control. Psychological Review, 108, 624-652.

Calvert, G., Spence, C., \& Stein, B. E. (Eds.) (2004). The handbook of multisensory processes. Cambridge, MA: MIT Press.

Davies, P. L., Segalowitz, S. J., Dywan, J., \& Pailing, P. E. (2001). Error-negativity and positivity as they relate to other ERP indices of attentional control and stimulus processing. Biological Psychology, 56, 191-206.

Dehaene, S., Posner, M. I., \& Tucker, D. M. (1994). Localization of a neural system for error detection and compensation. Psychological Science, 5, 303-305.

Desmedt, J. E., \& Robertson, D. (1977). Differential enhancements of early and late components of the cerebral somatosensory evoked potentials during forced-pace cognitive tasks in man. Journal of Physiology, 271, 761-782.

EIMER, M., \& Forster, B. (2003). Modulations of early somatosensory ERP components by transient and sustained spatial attention. Experimental Brain Research, 151, 24-31. 
Eimer, M., Forster, B., Fieger, A., \& Harbich, S. (2004). Effects of hand posture on preparatory control processes and sensory modulations in tactile-spatial attention. Clinical Neurophysiology, 115, 596-608.

Falkenstein, M., Hohnsbein, J., Hoormann, J., \& Blanke, L. (1991). Effects of crossmodal divided attention on late ERP components: II. Error processing in choice reaction tasks. Electroencephalography \& Clinical Neurophysiology, 78, 447-455.

Forster, B., EARDLEy, A. F., \& Eimer, M. (2007). Altered tactile spatial attention in the early blind. Brain Research, 1131, 149-154.

Forster, B., \& Eimer, M. (2004). The attentional selection of spatial and non-spatial attributes in touch: ERP evidence for parallel and independent processes. Biological Psychology, 66, 1-20.

Forster, B., \& EIMER, M. (2005a). Covert attention in touch: Behavioral and ERP evidence for costs and benefits. Psychophysiology, 42, 171-179.

Forster, B., \& Eimer, M. (2005b). Vision and gaze direction modulate tactile processing in somatosensory cortex: Evidence from eventrelated brain potentials. Experimental Brain Research, 165, 8-18.

García-Larrea, L., Lukaszewicz, A.-C., \& Mauguière, F. (1995). Somatosensory responses during selective spatial attention: The N120-to-N140 transition. Psychophysiology, 32, 526-537.

Gehring, W. J., Goss, B., Coles, M. G., Meyer, D. E., \& Donchin, E. (1993). A neural system for error detection and compensation. Psychological Science, 4, 385-390.

Holroyd, C. B., Dien, J., \& Coles, M. G. (1998). Error-related scalp potentials elicited by hand and foot movements: Evidence for an output-independent error-processing system in humans. Neuroscience Letters, 242, 65-68.

Kennett, S., Spence, C., \& Driver, J. (2002). Visuo-tactile links in covert exogenous spatial attention remap across changes in unseen hand posture. Perception \& Psychophysics, 64, 1083-1094.

Kopp, B., Rist, F., \& Mattler, U. (1996). N200 in the flanker task as a neurobehavioral tool for investigating executive control. Psychophysiology, 33, 282-294.

Liotti, M., WoldorfF, M. G., Perez, R., III, \& Mayberg, H. S. (2000). An ERP study of the temporal course of the Stroop color-word interference effect. Neuropsychologia, 38, 701-711.

Maravita, A., Spence, C., \& Driver, J. (2003). Multisensory integration and the body schema: Close to hand and within reach. Current Biology, 13, R531-R539.

MichIE, P. T. (1984). Selective attention effects on somatosensory eventrelated potentials. Annals of the New York Academy of Science, 425 , 250-255.

Michie, P. T., Bearpark, H. M., Crawford, J. M., \& Glue, L. C. T. (1987). The effects of spatial selective attention on the somatosensory event-related potential. Psychophysiology, 24, 449-463.

Nieuwenhuis, S., Yeung, N., VAn den Wildenberg, W., \& RidDERINKHOF, K. R. (2003). Electrophysiological correlates of anterior cingulate function in a go/no-go task: Effects of response conflict and trial type frequency. Cognitive, Affective, \& Behavioral Neuroscience, 3, 17-26.
Schürmann, M., Kolev, V., Menzel, K., \& Yordanova, J. (2002). Spatial coincidence modulates interaction between visual and somatosensory evoked potentials. NeuroReport, 13, 779-783.

Shore, D. I., Barnes, M. E., \& Spence, C. (2006). Temporal aspects of the visuotactile congruency effect. Neuroscience Letters, 392, 96-100.

Shore, D. I., \& Simic, N. (2005). Integration of visual and tactile stimuli: Top-down influences require time. Experimental Brain Research, 166, 509-517.

Spence, C., \& Driver, J. (Eds.) (2004). Crossmodal space and crossmodal attention. Oxford: Oxford University Press.

Spence, C., Pavani, F., \& Driver, J. (2004). Spatial constraints on visual-tactile crossmodal distractor congruency effects. Cognitive, Affective, \& Behavioral Neuroscience, 4, 148-169.

Spence, C., Pavani, F., Maravita, A., \& Holmes, N. (2004). Multisensory contributions to the $3-\mathrm{D}$ representation of visuotactile peripersonal space in humans: Evidence from the crossmodal congruency task. Journal of Physiology, 89, 171-189.

van VeEn, V., \& CARTER, C. S. (2002). The anterior cingulate as a conflict monitor: fMRI and ERP studies. Physiology \& Behavior, 77, 477-482.

VAn Velzen, J., Forster, B., \& Eimer, M. (2002). Temporal dynamics of liberalized ERP components elicited during endogenous attentional shifts to relevant tactile events. Psychophysiology, 39, 874-878.

Yeung, N., Botvinick, M. M., \& Cohen, J. D. (2004). The neural basis of error detection: Conflict monitoring and the error-related negativity. Psychological Review, 111, 931-959.

\section{NOTES}

1. Typically, visual distractors are presented at either the same hand as are tactile targets or at the other. In the present study, visual distractors were presented only from locations on the same hand as were tactile targets, since under these conditions visual distractor effects are maximal.

2. Early somatosensory modulations (i.e., in the time range of the P100 and N140 components) have been reported in numerous previous studies that have also employed linked earlobe references (e.g., Eimer \& Forster, 2003; Eimer, Forster, Fieger, \& Harbich, 2004; Forster, Eardley, \& Eimer, 2007; Forster \& Eimer, 2004, 2005a, 2005b; Michie, 1984; Michie et al., 1987; Van Velzen, Forster, \& Eimer, 2002), although single earlobes (Desmedt \& Robertson, 1977) and the nose (García-Larrea et al., 1995) also have been used as reference sites. Thus, the absence of any early somatosensory modulations in the present study is most likely not due to the use of linked earlobe references.

3. The absence of any attentional or perceptual effects on somatosensory processing is most likely not linked to data acquisition or analysis procedures. The authors have previously shown attentional (e.g., Forster et al., 2007; Forster \& Eimer, 2005a) and perceptual (e.g., Forster \& Eimer, 2005b) effects on early somatosensory processing when using similar experimental setups, EEG recording, and analysis procedures, and testing the same number of participants.

(Manuscript received July 17, 2006; revision accepted for publication December 9, 2007.) 